Erratum

\title{
Erratum to: Contribution to the improvement of heritage mural painting non-destructive testing by stimulated infrared thermography
}

Eur. Phys. J. Appl. Phys. (2013) 64: 11002

Jean-Luc Bodnar, Kamel Mouhoubi, Luigi Di Pallo, Vincent Detalle, Jean-Marc Vallet, and Thierry Duvaut

Published online: 21 November 2013 - (C) EDP Sciences 2013

When published on 8 October 2013, the article was erroneously attributed article number 11001 . Number has been corrected and proper citation of the article should consecutively read: Jean-Luc Bodnar, Kamel Mouhoubi, Luigi Di Pallo, Vincent Detalle, Jean-Marc Vallet, Thierry Duvaut, Eur. Phys. J. Appl. Phys. 64, 11002 (2013). 